\title{
Nature inspired optimization and its application to engineering
}

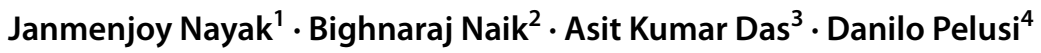 \\ Published online: 1 March 2021 \\ (c) The Author(s), under exclusive licence to Springer-Verlag GmbH, DE part of Springer Nature 2021
}

The nature which evolved billions of years ago provides a rich source of inspiration and interest among the researchers of various research community to develop a varied range of nature inspired optimization algorithms. Most of the realworld applications include optimization problems with high nonlinear constraints. To resolve those real-life problems, some of the earlier developed conventional optimization techniques such as gradient-based methods fails and most of the solutions of the real-life problems have been attained by using heat and trail methods. Therefore, to solve the highly nonlinear problems, nature inspired optimization algorithms are utilized. The success rate of these nature inspired algorithms is mainly due to their flexibility and capability in solving the NP-hard problems. Several algorithms have been developed by taking the inspiration of birds, insects, fish, ant, lion etc., which are the astonishing creatures of nature. The nature inspired optimization techniques provide adaptive computational tools for the complex optimization problems and diversified engineering applications. As nature inspired algorithms are simple and versatile in nature, many researchers in the world have always been enamored towards the development of some advanced intelligent algorithms and its applicability in solving wide range of complex engineering problems efficiently.

This Special issue includes some of the interesting applications of nature inspired optimization algorithms in

Janmenjoy Nayak

mailforjnayak@gmail.com

1 Department of Computer Science and Engineering, Aditya Institute of Technology and Management (AITAM), Tekkali, Srikakulam, A.P., India

2 Department of Computer Application, Veer Surendra Sai University of Technology, Burla, Odisha Sambalpur-768018, India

3 Department of Computer Science and Technology, Indian Institute of Engineering Science and Technology(IIEST) Shibpur, Howrah, West Bengal, India

4 Communication Sciences, CosteSant'agostino Campus Teramo, University of Teramo, Teramo, Italy resolving nonlinear complex problems such as automobile, insurance, fraud detection, using fuzzy clustering algorithm based on modified whale optimization algorithm, Image compression using explored bat algorithm by Renyi 2-d histogram based on multilevel thresholding, MC/DC guided Test Sequence Prioritization using Firefly Algorithm, accurate localization of sensor nodes in underwater sensor networks using a Doppler shift and modified genetic algorithm based localization technique, Differential evolution algorithm tuned tilt integral derivative controller with filter controller for automatic generation control, Parameters tuning of a quadrotor PID controllers and cryptanalysis of S-DES using nature inspired algorithm etc. Moreover, the efficiency of these algorithms in various diversified application such as a unified intrusion detection system for IoT environment, modified crash-minimization path designing approach for autonomous material handling robot, software fault localization using BP neural network based on function and branch coverage, a novel stacked sparse denoising autoencoder for mammography restoration to visual interpretation of breast lesion, ensemble learning with recursive feature elimination integrated software effort estimation, hyper-parameter tuned deep Q network for area estimation of oil spills, multiagent path planning with nonlinear restrictions have also been analyzed.

Sahu et.al has introduced an optimal differential evolution (DE) approach for AGC of five areas multi-element re-heat thermal power models with nonlinearities such as generator rate constraint (GRC) and governor dead band (GDB). Being a heuristic optimization technique, DE has been chosen to be the control parameter to manage the parameters such as crossover possibility as well as step size for attaining a better model using multiple iterations analytically by the authors. Additionally, proportional-integral (PI)/proportional integral derivative (PID)/ tilt integral derivative with filter (TIDF) type controller methods have been customized by the authors and their growth has been optimized by the DE algorithm respectively.

Pattanayak and Choudhury have designed a modified crash minimization path for independent material managing 
robots. The potentiality of artificial potential fled (APF), particle swarm optimization (PSO), and Improved PSO (IPSO) advances has been utilized by the authors for creating and designing the better potential optimum trajectory for a material handling robot. The authors have used optimization techniques to prompt the optimum track such as smooth and crash-free through restrain length, travel time, and computational time amid source as well as a destination point. Using the three approaches made it easy to illustrate the difficult environmental surroundings.

Majhi has introduced a 2-stage fraud recognition model that utilizes outlier extraction by using a Whale optimization algorithm (WOA). The stochastic optimization algorithm (WOA) was utilized to discover the overall optima of a presented dataset. The authors have proposed a fuzzy clustering technique by utilizing both FCM as well as Modified WOA (MWOA). The Optimization technique has been used to extract the inadequacy of the FCM.

Kumar et.al has introduced a new unified intrusion detection (UID) model for IoT surroundings to protect the networks. The authors have considered UNSW-NB15 as a standard dataset for recognizing malicious actions in the network. The decision tree (DT) models have been trained by using 13 attributes of the dataset. The projected model was found to have a higher rate compared to Dendron as well as ENADS.

Hassani et.al has aimed to examine the power of a quadrotor by PID controller. Due to irregularities, pairing, and under-actuation limitations, the method inflicts complexities to produce its regulator by using standard ways. So, natureinspired algorithms (NIA) such as Evolution Strategies, Genetic Algorithm (GA), Differential Evolutionary (DE), and Cuckoo Search optimization (CSO) have been used to attain the optimal path tracking. The proposed optimization techniques were utilized to optimize the controllers without departing throughout the linearization.

Manohar et.al has investigated Bat Algorithm (E-BA) with a $2 \mathrm{D}$ histogram which is relied on multi-level image thresholding for the firmness of the image by exploiting the Renyi entropy for efficient image thresholding. The identical allocation of energy above the entire 1D histogram made it computationally difficult. So, an optimization technique called the Bat algorithm has been introduced by the authors to enhance the image value of a renovate illustration known as a 2-D histogram-based multilevel thresholding to increase the entropy of Renyi.

Maru et.al has introduced an efficient advance for liability localization using a Backpropagation neural network (BPNN). A novel feature matrix was projected by the authors to train the BPNN technique. The proposed model comprises both functions as well as branch reporting data of several test cases. It was found using the EXAM_Score evolution metric to determine the efficiency of the anticipated fault localization method.

Barisal et.al has used the Firefly algorithm (FA) which is an NIA with an ad hoc objective function to produce the finest test paths directed by the Decision Coverage (or) Modified Condition (MC/DC) principle in the form of a channeled matrix. As it is difficult to attain satisfactory outcomes on the cohort of non-surplus as well as optimized test sequences, the authors have found FA as an efficient optimization technique for the regression testing and to afford better outcomes respectively.

Datta and Dasgupta have proposed a Doppler shift based localization pursued by GA based optimization method that enhances accurateness in restricting unidentified nodes in underwater antenna networks. The projected technique predicted sink nodules as a major technique in considering bulk computational loads on the report of being relatively more available and useful as contrasted to any other nodes in the network. The major aim of an optimization technique is to know the overall distance enclosed to inclusive the journey. So, being an optimization technique, GA has efficiently modified and utilized to attain better outcomes.

Ghosh et.al has introduced a new stacked sparse denoising auto-encoder (SSDAE) for mammography reinstatement to image explanation of breast lesion. The authors have designed a deep-stacked convolutional auto-encoder by merging the auto-encoder and the de-convolution network that conjointly lessens loud objects as well as enhances image information in a mammogram. It was found measuring multiple loss task to make reach optimization sooner in convolutional technique throughout the training periods. Ultrasonographic and $\mathrm{X}$-ray mammograms were used for the legalization of the model.

Rao and Rao have proposed a new advance using ensemble learning (EL) with recursive quality elimination to measure the effort. The authors have tested the projected method using seven ML classifiers. Based on the consequences of recursive feature elimination, the characteristics are being graded and the finest features are input to the next model based on EL. Compared to other techniques, ML-based advances are intense to measure or calculate the effort more precisely because of their active adaptivity with any kind of data.

Kamal et.al has proposed a method of the cryptanalysis of Simplified Data Encryption Standard (S-DSE) by utilizing NIOA. As S-DSE is a NP-Hard problem, the authors have examined the S-DES through NIOA such as CSO, FA, and Black-Hole Optimization Algorithms (BHOA) and developed the intrinsic individuality of such occurrence to resolve optimization issues. A relative study was presented on the effectiveness of the three projected optimization techniques with the earlier recognized GA as well as Memetic 
Algorithms. The proposed model outperformed with better results than other compared methods.

Banerjee et.al has introduced Gamma-Levy Hybrid Metaheuristic with Conditional Evolution (GLHM-CE) technique. The proposed method is known to be a metaheuristic algorithm that is utilized to develop a dispersed manage approach for a group of unmanned aerial vehicles for fast detention and inference of spill boundary. Each agent was found to be managed by a Deep Q Network (DQN) whose Hyper-Parameters (HP) is tuned by GLHM-CE. Due to having less time complexity as well as convergence time, the optimization techniques were found to be a better choice compared to other methods.

Marques et.al has presented a new basic mathematical technique that can resolve a path planning issue including three controllers in a triangular configuration. The difficulty is formed to optimize the lines of the agents and to reduce the traveled distance. The trajectories are attained in a way that diverges them from permanent obstacles whose sizes are recognized. Also, geometry and direction restraints of the formation of the multi-agent are made compulsory. So, optimization techniques such as GA, DE, and PSO are sued to resolve the nonlinear equations that signify the model. PSO was found presenting the low processing instance for every simulated case.

This special issue also highlights some challenges such as stability of nature inspired algorithms, theoretical framework of parameter tuning and control, solutions of large scale real-life applications and progress on dealing non deterministic NP problems in order to inspire more research and to provide insight into different nature inspired algorithms among the readers and researchers.Being the guest editors, we anticipate that the spectrum of research covered in this special issue will be of an asset for an entire host of readers/ researchers working in the sphere of nature inspired optimization algorithms. We have tried the best to maintain the balance between the types of articles including the methods and their application domain. We are indebted to the authors of this issue for their valued research contributions and supportive towards the spirit for changing the paper as per the reviewer's comments. The technical standards and quality of published articles in this special issue are based on the strength and expertise of the reviewer board members who have been grossly involved in providing high-quality reviews for the submitted papers. Also, we are grateful to the editorial members and Editor in Chief for their constant support and cooperation in all possible ways for the successful completion of this issue.

Publisher's Note Springer Nature remains neutral with regard to jurisdictional claims in published maps and institutional affiliations. 